\title{
Unsteady Pressure Field Investigation of an Axial Fan-Blade Unsteady Pressure Field Measurement
}

\author{
C. Xu and R. S. Amano \\ Department of Mechanical Engineering, University of Wisconsin-Milwaukee, Milwaukee, \\ Wisconsin, USA
}

A $1.829 \mathrm{~m}$ (6 ft) diameter industrial large flow-rate axial fan operated with $1770 \mathrm{rpm}$ was studied experimentally in laboratory conditions. The flow characteristics near the fan blade surfaces were investigated by measuring the pressure distributions on the blade suction and pressure surfaces and the results were discussed by comparing with analytical formulations. Flow visualizations were also performed to validate the flow characteristics near the blade surface and demonstrated that the flow characteristics near the fan blade surface were dominated by the centrifugal force of the fan rotation, which result in strong three-dimensional flows. The time-dependent pressure measurement showed that the pressure oscillations on the fan blade were significantly dominated by vortex shedding from the fan blades. It was further demonstrated that the pressure distributions during the fan start-up were severely unsteady, and the main frequency variation of the static pressure was much smaller than the fan rotational frequency. The time-dependent pressure measurement when the fan operated at a constant speed showed that the magnitude of the blade pressure variation with time and the main variation frequency was much smaller than the fan rotational frequency. The pressure variations that were related to the vortex shedding were smaller than the fan rotational frequency. The complete set of blade-surface pressure measurements obtained can be used as a guide for performance improvements, vibration analysis, and CFD code validation.

Keywords Unsteady aerodynamic; Pressure measurement; Flow visualization; Axial fan

Received 25 August 2000; accepted 2 August 2001.

Address correspondence to Ryoichi S. Amano, Mechanical Engineering Department, University of Wisconsin-Milwaukee, EMS Building, P. O. Box 784, Milwaukee, WI 53201.
Detailed experimental research in the axial fans has been one of the major concerns of HVAC industries. This is because a significant improvement in the axial fan performance can be achieved if the aerodynamic and vibration losses are reduced within the fan. Moreover, a better understanding of axial fan blade flow and its pressure characteristics help lead to improved fan performance, thereby improving the fan design, can contribute to reduced operating costs. The prediction of the fan flow characteristics using Computational Fluid Dynamics (CFD) technology is becoming available in industries. The development of the unsteady three-dimensional CFD codes for fan design has become more important. The validation of the CFD codes needs detailed pressure measurements, especially the pressure information on the fan blade surfaces.

Fan industries have been developing large flow-rate fans for many years. Nevertheless, they still face many difficulties in constructing efficient and stable fan units. This is primarily because the increase of flow-rate sacrifices the fan stability. To solve these problems, an extensive unsteady aerodynamic study is in high demand. Because the fan efficiency and corresponding loading are caused by an aerodynamic behavior which relates to the fan performance, the underlying fluid dynamics must be well understood to accurately determine the structures and to improve fan efficiency. Earlier such research on fans appeared almost three decades ago. However, due to the limitations of the instrumentation, measurement was limited to steady performance studies. Abramovich (1963) studied the air distribution in a dead-end channel supplied by axial fan. His study showed that in a duct system, it resulted in very high velocities at discharge but relatively low velocities throughout the body of the opening. Recently, Mutama and Hall (1996) studied the pressure distributions of a jet fan traveling from one wall along the axial direction of a tunnel in order to optimize the positioning of the jet fan. Experimental and theoretical studies have been reported by several researchers (Tsurusaki, 1997; Kaupert and Staubli, 1999a, 1999b) to improve fan and pump design. However, due to the limitations of the measuring devices and the high cost of the facility, most of the fan tests were focused on the 
velocity fields and inlet/outlet pressure-field studies based on a small fan model. The cross-fan is a popular topic in fan research because it could be easily installed in a small wind tunnel and it is easy to set up the testing devices, such as pressure and velocity transducers and other instruments in inlet and outlet sections. Although most of the measurements were still limited to velocity and pressure fields at a steady condition, recently some unsteady measurements and theoretical analyses on the pumps and axial-flow impellers were reported (Qin and Tsukamoto, 1997; Myers, Ward, and Bakker, 1997). However, only a few studies have been related to unsteadypressure blade measurements. Because of the limitations of most research laboratories and the relatively high cost of large axial fan units, there is almost no open literature available on studies of unsteady pressure measurements in a large-size axial fan.

The main source of the vibration in fans is due to rotational imbalance caused by the fan rotor and the aerodynamic force due to unsteady airflow. In the case of fan rotational imbalance, because most of the fan units have a relatively short axial shaft length, the only likely form of the imbalance to give instability is by a static imbalance. This arises from the lack of coincidence between the center of gravity of the impeller and the axis of rotation, which provides an unbalanced moment. With the development of balancing technology, the fan vibration due to imbalance can be reduced to a rather small level. On the other hand, the fan vibration due to the fan aerodynamic force still cannot be well resolved, which requires aerodynamics studies of the large airflow volume fan.

Ability to make an unsteady measurement in a complicated geometry greatly enhances the investigative efforts of researchers to understand the behavior of fan aerodynamics. There have been a number of experimental studies reported on the axial machine (Gallus, Lambertz, and Wallmann, 1980; Dring et al., 1982; Capace and Fleeter, 1989). Most of the studies dealt with the rotor-stator interaction through the velocity field and wake characteristics when an axial machine rotated at a constant speed. More recently, Huyer, Simms, and Robinson (1996) studied wind turbine unsteady aerodynamics associated with yaw angle change through the steady pressure measurement in which some analyses were also performed regarding unsteady aerodynamics. However, no studies dealt with large unsteady flow characteristics. In recent years, substantial developments have been made toward improvements in the quality of support facilities available for research in experimental aerodynamics, especially due to the development of pressure transducer manufacturing technology and the improved capability of PC-based data acquisition systems. For example, Kaupert and Staubli (1999a, 1999b) extensively investigated the unsteady pressure field within a centrifugal pump impeller. The present work is aimed at the unsteady aerodynamic study of an axial fan by providing some fundamental data on the subject. The main objectives of this study are to investigate unsteady pressure on the fan blade surface during the period between the fan start-up and acceleration to its operating speed as well as fan operating at a constant rotational speed.

In this paper, detailed unsteady pressure behaviors of the fan blade are reported. Discussions of the unsteady aerodynamics produced by an axial fan can provide increased understanding of the unsteady flow-fields generated by other rotational machines, such as submarine propellers, helicopter rotors, and first-stage fans of compressors.

\section{TEST FACILITY AND INSTRUMENTATION}

An eight-blade industrial axial fan which was designed for ventilation and oil industries was studied as shown in Figure 1. The outer diameter of the blades is $d_{2}=1.829 \mathrm{~m}(6 \mathrm{ft})$ and the

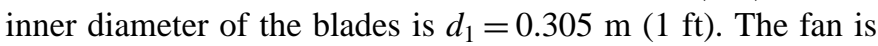
installed at the axial center of a streamline-shaped casing with a casing length of $0.37 \mathrm{~m}$ and a diameter of $1.84 \mathrm{~m}$ at the inlet of the casing. The fan blade stagger angle from the rotation plan is $30^{\circ}$. The fan blade was designed as a constant chord along the radial direction. The length of the chord is $C=0.246 \mathrm{~m}$. The steady rotational speed is $\omega=1770 \mathrm{rpm}$ with the tip velocity of the blade $u_{t} \approx 169.5 \mathrm{~m} / \mathrm{s}$. A dynamometer was used to verify the rotational speed. The laboratory temperature was about $20^{\circ} \mathrm{C}$ and the Reynolds number based on the blade diameter was in the order of $10^{6}$ at the rotating speed of $\omega=1770 \mathrm{rpm}$. A specially made slip-ring was mounted on the fan shaft to transfer the acquired pressure transducer signals from the rotational blade to a computer data acquisition system. The slip-ring was calibrated in the factory, the uncertainty of the voltage was less than $\pm 0.25 \mathrm{mV}$, and the time delay was less than $2 \mu$ s with $95 \%$ confidence. The overall unsteady pressure measurement system with measurement uncertainty of pressure and time delay with 95\% confidence are $(Y+351.1 P a, Y+287.4 P a)$ and $(5.39 \mu \mathrm{s}$, $15.23 \mu \mathrm{s})$ respectively as reported by Xu and Amano (1999). These uncertainties are less than $10 \%$ of the minimum pressure. The eigenfrequency of the pressure transducer in air was near $500 \mathrm{kHz}$, which was well above the frequencies of interest - a maximum of $2 \mathrm{kHz}$.

The low pass filter frequency set is one of the important factors of the measurement system. The common method is to let

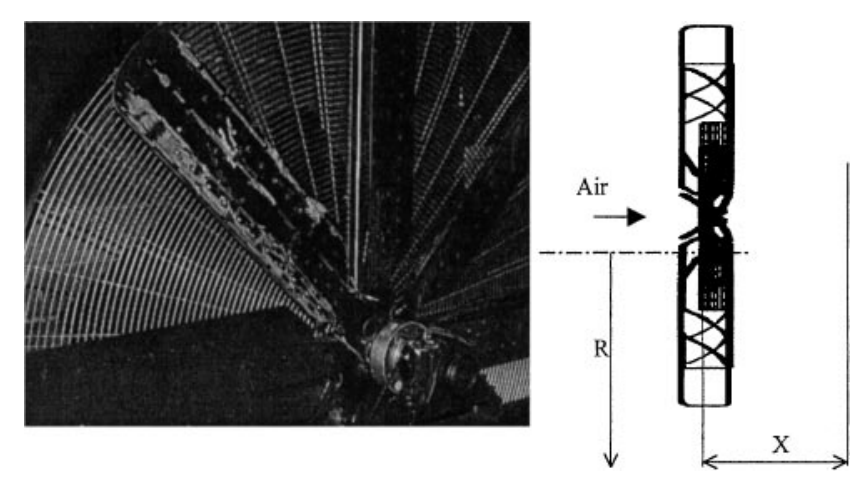

FIGURE 1

Test fan and its side view. 


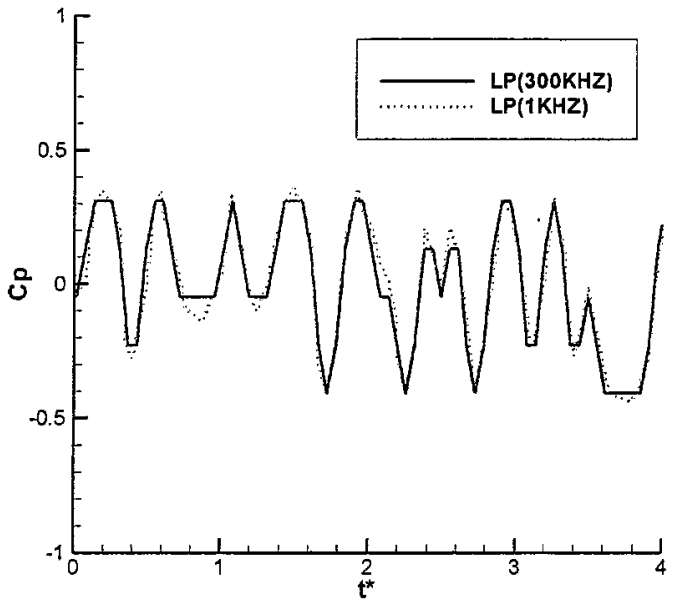

FIGURE 2

LP filter frequency influence.

the LP filter frequency equal the Nyquist frequency in order to minimize the digitization rollback error. According to the fan's steady rotation speed, the measurement of LP frequency is set for $1 \mathrm{kHz}$ for unsteady blade pressure measurement, which has the highest sample frequency. However, due to the limitation of the computer hard drive and large amount of data, the LP filter frequency is set to $300 \mathrm{~Hz}$, which is acceptable for industrial applications and CFD code errors. The comparison of using different LP filter frequencies is shown in Figure 2. It is shown that an LP filter set to $300 \mathrm{~Hz}$ can obtain reasonable results. The dynamic pressure data $\mathrm{P}$ were collected at a sample rate of $1 \mathrm{kHz}$ and alias filtered at about $300 \mathrm{~Hz}$ in this study.

To quantify the effect of the measured drift and repeatability with the bias error, three sets of the test data for each position were collected and compared. Various procedures were used to ensure the measurement system by maintaining the required accuracy levels. The signal analysis, when the fan is in steady operation, was based on a sampling set of $2^{15}$ points with a sample frequency of about $1 \mathrm{kHz}$, slight higher than that at the actual fan rotation of $966 \mathrm{~Hz}$. For the fan starting data analysis, a sample set of $2^{18}$ with sample frequency about $0.5 \mathrm{kHz}$ was used. Phase averaging of the unsteady measurement is a common practice in unsteady measurements (Minniti, Sullivan, and Mueller, 1998) to eliminate a part of the signal linked to stochastic effects and to reduce the data size for signal interpretation of the rotational harmonics. The signals were phase averaged in this study. For representing the variations of the unsteady pressure distributions of the fan identically, a smoothing technique provided by a Matlab signal processing toolbox (1996) was used.

The measured pressure is non-dimensionalized using the outlet reference averaged total pressure and static pressure. The reference total pressure $p_{\text {ref }}^{*}$ and static pressure $p_{\text {ref }}$ were measured at the station $\left(X_{\text {ref }}=0.186, R_{\text {ref }}=0.6\right)$ and $p_{\text {ref }}{ }^{*}-p_{\text {ref }}=$ 824.8 Pa. This reference location is near the geometrical center of the blade. The pressure coefficient was given by:

$$
C_{p}=\frac{p}{p_{\text {ref }}{ }^{*}-p_{\text {ref }}}
$$

Sixty test positions were used on the blade suction and pressure surfaces. The location coordinates on the suction surface $(i=1$ to 4 ) and pressure surface ( $i=5$ to 8 ) are listed in the Table I where $i$ and $j$ indices indicate the chord-wise direction and radial directions, respectively. All the pressure measurement data locations listed in Table I are available in the Fluid Dynamic Research Center measurement database. The discussions in this paper are focused on the locations of interest.

\section{BLADE SURFACE FLOW VISUALIZATION}

The blade-surface flow visualization method used in this study is based on a well-established technique presented by Huntsman and Hodson (1995) for both two- and three-dimensional flow studies. It is commonly recognized that, although important and useful, these flow patterns only provide information about the nature of the near surface flow. Perspective photographs of the suction surface near the hub and tip parts are shown in Figures 3 and 4, respectively. The main flow directions on both parts are toward the blade tip direction due to the centrifugal force during fan rotation. The flow pattern near the hub seems to be mainly influenced by both the centrifugal force and the blade stagger angle as shown in Figure 3. The

TABLE I

Locations of the testing points ( $\left.Y_{\text {ref }}, R_{\text {ref }}\right)$

\begin{tabular}{lcccccccc}
$j i$ & 1 & 2 & 3 & 4 & 5 & 6 & 7 & 8 \\
\hline 1 & $(0.071,0.945)$ & $(0.304,0.945)$ & $(0.611,0.945)$ & $(0.927,0.945)$ & $(0.071,0.945)$ & $(0.304,0.945)$ & $(0.611,0.945)$ & $(0.927,0.945)$ \\
2 & $(0.071,0.882)$ & $(0.304,0.862)$ & $(0.611,0.862)$ & $(0.927,0.882)$ & $(0.071,0.862)$ & $(0.304,0.928)$ & $(0.611,0.928)$ & $(0.927,0.882)$ \\
3 & $(0.071,0.797)$ & $(0.304,0.779)$ & $(0.611,0.779)$ & $(0.927,0.797)$ & $(0.071,0.781)$ & $(0.304,0.845)$ & $(0.611,0.845)$ & $(0.927,0.781)$ \\
4 & $(0.071,0.630)$ & $(0.304,0.611)$ & $(0.611,0.611)$ & $(0.927,0.630)$ & $(0.071,0.615)$ & $(0.304,0.666)$ & $(0.611,0.666)$ & $(0.927,0.615)$ \\
5 & $(0.071,0.465)$ & $(0.304,0.528)$ & $(0.611,0.528)$ & $(0.927,0.465)$ & $(0.071,0.445)$ & $(0.304,0.508)$ & $(0.611,0.508)$ & $(0.927,0.445)$ \\
6 & $(0.071,0.382)$ & $(0.304,0.444)$ & $(0.611,0.444)$ & $(0.927,0.382)$ & $(0.071,0.365)$ & $(0.304,0.410)$ & $(0.611,0.410)$ & $(0.927,0.365)$ \\
7 & $(0.071,0.298)$ & $(0.304,0.361)$ & $(0.611,0.361)$ & $(0.927,0.298)$ & $(0.071,0.282)$ & $(0.304,0.333)$ & $(0.611,0.333)$ & $(0.927,0.282)$ \\
8 & & $(0.304,0.278)$ & $(0.611,0.278)$ & & & $(0.304,0.267)$ & $(0.611,0.267)$ & \\
\hline
\end{tabular}




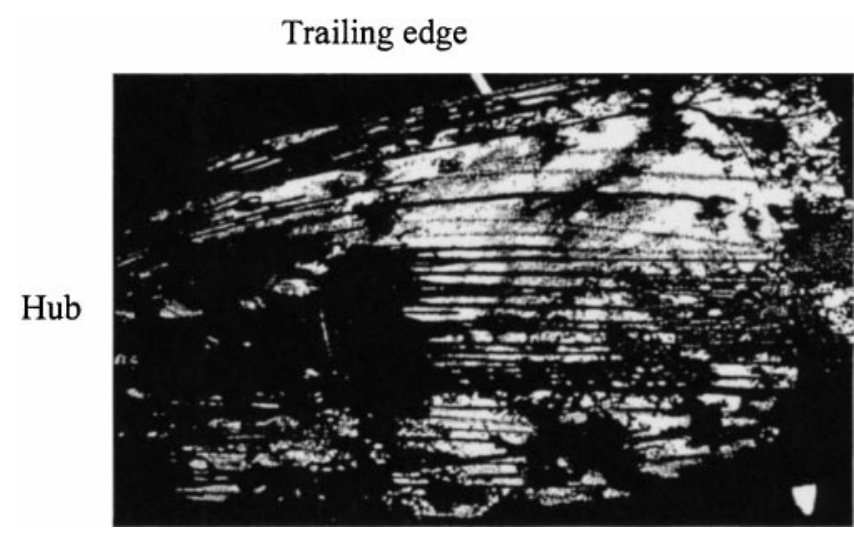

FIGURE 3

Flow pattern on the suction surface near the hub.

flow patterns near both leading and trailing edges have similar characteristics. Contrastingly, the flow pattern near the tip of the blade is significantly different from the flow pattern near the hub, as shown in Figure 4. The streak lines slightly tilt towards the trailing edge in the suction surface. This effect may be due to the flow acceleration on the blade suction surface. Because the large tangential velocity strongly influences the flow field near the tip, the flow severely accelerates near the tip. The flow visualization photographs of the pressure-surface near the hub and tip sides are also shown in Figures 5 and 6, respectively. Similar flow patterns are shown in these figures, too. It seems that the flow patterns on the pressure side are mostly influenced by two mechanisms: the centrifugal force and the stage angle of the blade. On the other hand, the tangential velocity on a blade surface seems to have little influence on the pressure side of the blade. This is because the magnitude of the tangential velocity is affected to the geometry of the blade. The secondary flows on both the suction and pressure surfaces were observed to be highly three-dimensional. The comparison of the flow visualizations between the suction and pressure surfaces shows that the difference of the blade shape in suction surface has some influence on the near blade flow, but has little influence on the

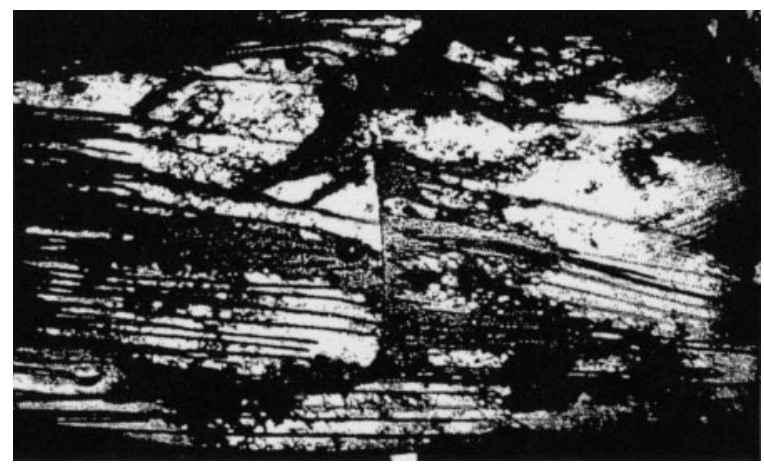

FIGURE 4

Flow pattern on the suction surface near the tip.

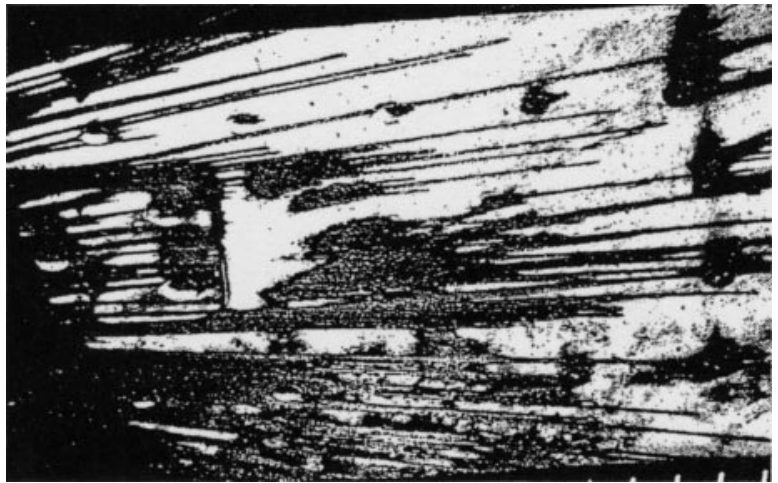

FIGURE 5

Flow pattern on the pressure surface near the hub.

near blade flow on the pressure surface. The near-blade flow characteristics are dominated by the centrifugal effect.

\section{THEORETICAL CONSIDERATIONS}

To analyze the unsteady pressure field, a vortex singularity method (Xu and Yeung, 1998) should be used. Assuming that the flow is two-dimensional within the same radial plan, consider the velocity induced by the vortices at the point $(r, \theta)$ in the stationary coordinate system. If the fan-blade-bounded vortex strength is $\gamma$, the velocity field around the fan blade can be expressed in a Fourier series as:

$$
\begin{aligned}
V_{r b}= & -\frac{Z}{2 \pi r} \int_{s 1}^{s 2}\left\{\sum_{n=1}^{\infty}\left[r_{b} / r\right]^{n Z} \sin \left[n Z\left(\theta-\theta_{b}-\omega t\right)\right]\right\} \gamma d s \\
V_{\theta b}= & -\frac{Z}{2 \pi r} \int_{s 1}^{s 2}\left\{1+\sum_{n=1}^{\infty}\left[r_{b} / r\right]^{n Z} \cos \left[n Z\left(\theta-\theta_{b}-\omega t\right)\right]\right\} \\
& \times \gamma d s
\end{aligned}
$$

Equations (2) and (3) show that the velocity variations due to the bounded vortices are dominated by the fan rotational speed. The velocity induced by the vortices shed from the fan blade trailing edge $\gamma_{w}$ can be expressed as (Xu and Yeung, 1998):

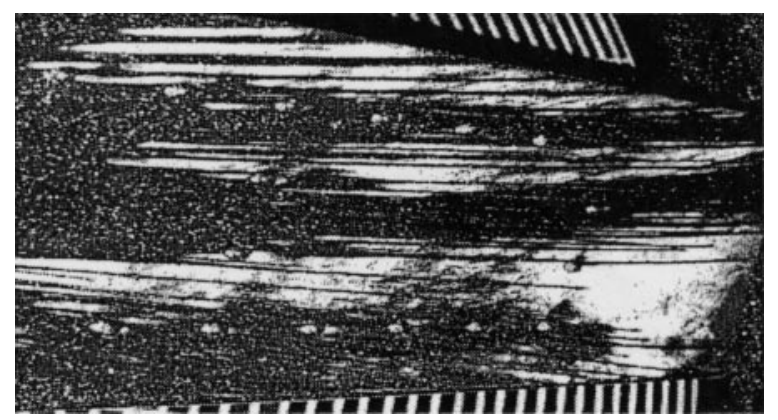

FIGURE 6

Flow pattern on the pressure surface near the tip. 


$$
\begin{aligned}
V_{r w}= & -\sum_{q=1}^{z} \int_{s 2}^{\infty} \frac{1}{2 \pi r} \frac{\left(r_{q} / r\right) \sin \left(\theta-\theta_{q}\right)}{1+\left(r_{q} / r\right)^{2}-2\left(r_{q} / r\right) \cos \left(\theta-\theta_{q}\right)} \\
& \times \gamma_{w q} d s \\
V_{\theta w}= & -\sum_{q=1}^{z} \int_{S 2}^{\infty} \frac{1}{2 \pi r} \frac{\left(r_{q} / r\right) \sin \left(\theta-\theta_{q}\right)}{1+\left(r_{q} / r\right)^{2}-2\left(r_{q} / r\right) \cos \left(\theta-\theta_{q}\right)} \\
& \times \gamma_{w q} d s
\end{aligned}
$$

According to the Helmholz theorem, the conservation of vortices leads to the conclusion that the rate change of total circulation around the fan is equal to the total shed vortices from the blade trailing edge per unit time. The strength of the vortices shed from the blade trailing edge can be expressed as:

$$
\gamma_{w q}=-1 / V(s) \frac{d}{d t} \int_{s 1}^{s 2} \gamma[t-\tau(s)] d s
$$

where $\tau(s)$ is given by:

$$
\tau(s)=\int_{s 1}^{s 2} 1 / V(s) d s
$$

Equations (4) and (5) indicated that the shed vortices induced velocities are changed with time according to their tangential distance $\left(\theta-\theta_{q}\right)$. The velocity field near the blade surface is the sum of the three types of induced velocity: incoming-flow velocity, blade bounded vortex induced velocity, and velocity induced by shed vortices, which can all be expressed as:

$$
\begin{aligned}
& V_{r}=V_{r \infty}+V_{r b}+V_{r w} \\
& V_{\theta}=V_{\theta \infty}+V_{\theta b}+V_{\theta w}
\end{aligned}
$$

The total velocity is then expressed as:

$$
V=V_{r}+V_{\theta}
$$

The main oscillating sources of the velocity field are induced velocities of bounded vortices (Equations (2) and (3)) and shed vortices (Equations (4) and (5)). The unsteady pressure can be calculated using the following unsteady Bernoulli's equation:

$$
p / \rho+V^{2} / 2+\partial \phi / \partial t-\left(p_{\infty} / \rho+V_{\infty}^{2} / 2+\partial \theta / \partial t\right)=g H
$$

where:

$$
\partial \theta / \partial t=Z \omega \gamma d s / 2 \pi \sum_{q=1}^{\infty}\left(r / r_{b}\right)^{-q Z} \cos \left[q Z\left(\theta-\theta_{b}+\omega t\right)\right]
$$

Equation (11) shows that the oscillation in the velocity field will cause the oscillation in the pressure field with time as well. The analysis shows that the two main sources; i.e. the blade-bound vortices and shed vortices, will cause two types of oscillations. The pressure variation frequencies are determined by those two types of velocity variation frequency.

\section{UNSTEADY PRESSURE MEASUREMENTS}

\section{Pressure Distributions When Fan Starts Up}

The fan start-up process consists of a strong interaction between the fan blade and air. The air flow from a non-moving state changes to a moving state during this stage. This massive turbulent process causes a large variation in the pressure, which will induce high vibration in the fan unit. The typical pressure variations with time for four different chord positions are shown in Figures 7-10. As shown in these figures, the pressure measurements show that the pressure variations with time have similar characteristics along the radial directions. Figure 7 shows that the pressure behavior near the leading edge has slightly different characteristics along the radial direction near the hub and tip. The pressure reaches the minimum level faster near the tip region than near the hub region. The minimum pressure near the tip is slightly higher than the tip region because the casing restricts the expansion of the air near the tip region. On the other hand, the flow near the hub has little restriction due to the design of the fan (in this fan unit, there is a casing to install the motor belts which blocks the flow-field near the hub region). The pressure distributions along the chordwise direction, $y / c=0.304,0.611$, and 0.927 , are shown in Figures 8, 9, and 10, respectively. The pressure distributions at these locations all have similar trends. The minimum pressure differences become smaller with the increase of the chord position. This is evidenced by the fact that, with the increase of the chord distance, the acceleration of the flow field decreases. The restraint of the casing and the low-speed air in the hub center become relatively small. The pressure distributions show that the final steady pressures decrease with the increase of the chord distance because the flow accelerates with the increase of the chord distance.

The pressure variations with time on the pressure surface of the blade with the chordwise distance $y / c=0.071,0.304$, 0.611 , and 0.927 are shown in Figures 11-14, respectively. The general trends are similar to those of the suction surface. The pressure level drops with time and reaches its minimum at about $t^{*}=6000$, then returns to the original level at about $t^{*}=$ 12000 (steady state). It is noted that the minimum pressure levels are higher near the tip than those on the suction surface. This is because the air flow acceleration on the pressure surface is much smaller than the suction surface near the tip region. Along the chordwise locations near the trailing edge, the minimum pressure levels are similar to those on the suction surface. Two flow streams of suction and pressure surfaces mix after the flow passes through the trailing edge. In general, the steady pressure levels are higher than those at similar chordwise locations on the suction surface. It is noteworthy that the pressure variation near the leading edge on the hub region has little variation. The 


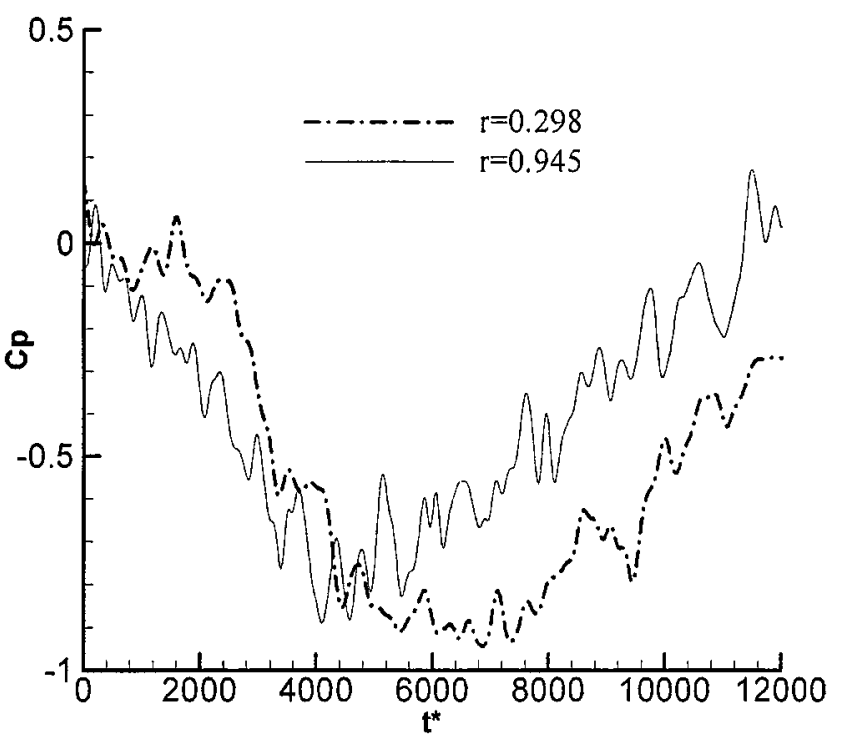

FIGURE 7

Unsteady pressure distributions on the suction side when fan starts up $(y / c=0.071)$.

magnitudes of the flow velocity both in chordwise and tangential directions are very low. The flow velocity does not change much during the fan starts up. The unsteady pressure variation frequencies both in suction and pressure surfaces were dominated by the fan-blade-bounded vortices as well as vortex shedding, as expressed in Equation (11). The main pressure variations with time were dominated by the fan rotational speed. Therefore, the unsteady pressure variations have similar frequency. However, the pressure variations with time take place much more slowly than the fan rotational frequency. It is shown that the pressure

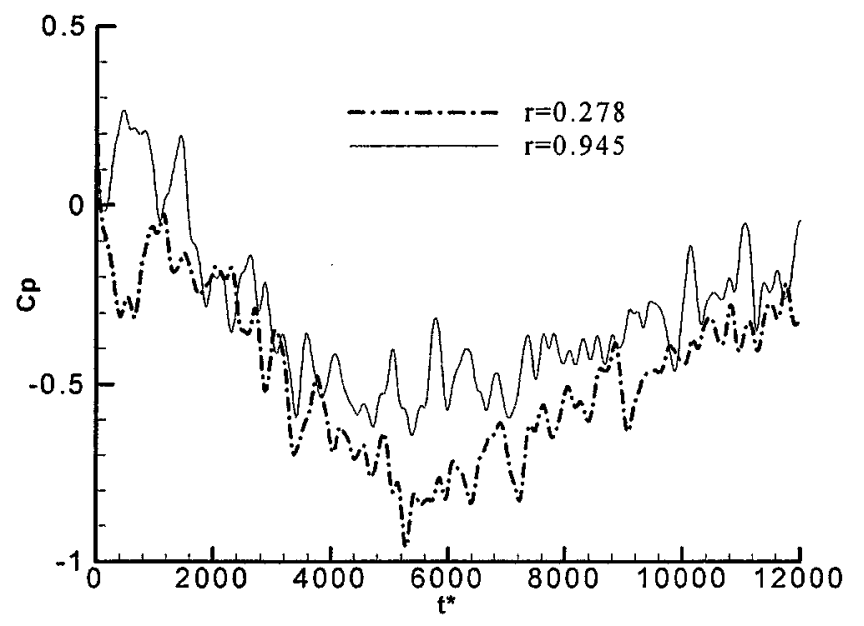

FIGURE 8

Unsteady pressure distributions on the suction side when fan starts up $(y / c=0.304)$.

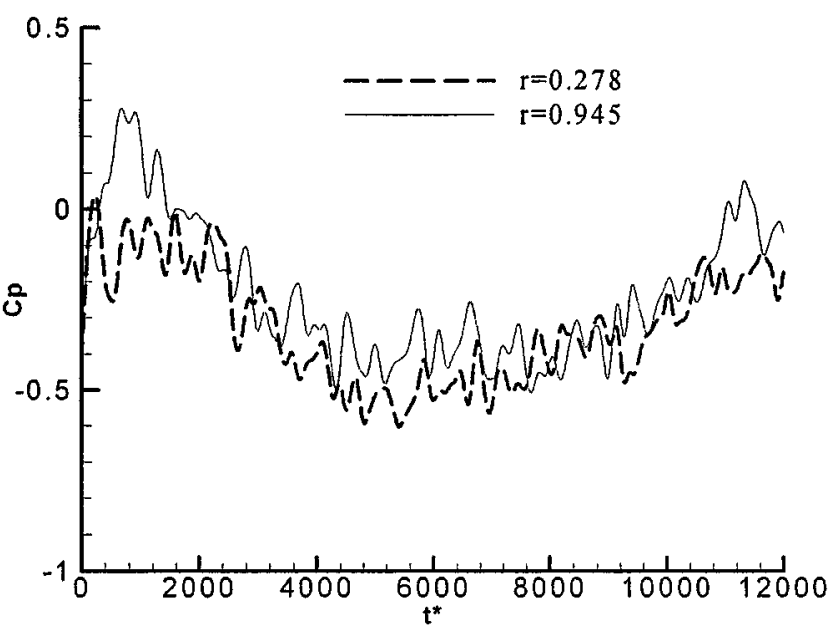

FIGURE 9

Unsteady pressure distributions on the suction side when fan starts up $(y / c=0.611)$.

reaches a steady state at about $t^{*}=12000$. However, the fan reaches its constant rotational state after $t^{*}=5000$.

\section{Pressure Distributions When Fan Runs at Constant Speed}

Most of the pressure field and velocity field measurements (Myers, 1997; Mutama and Hall, 1995) reported on the axial impeller or fan outlet were focused on the steady flow pressure distribution measurements. Due to the limitations of the measurement facility, transducer technology, and the cost of the large flow-rate fan device, little open literatures exists on the unsteady pressure measurements based on a large flow-rate axial fan. To advance our knowledge of the large flow-rate fan design and

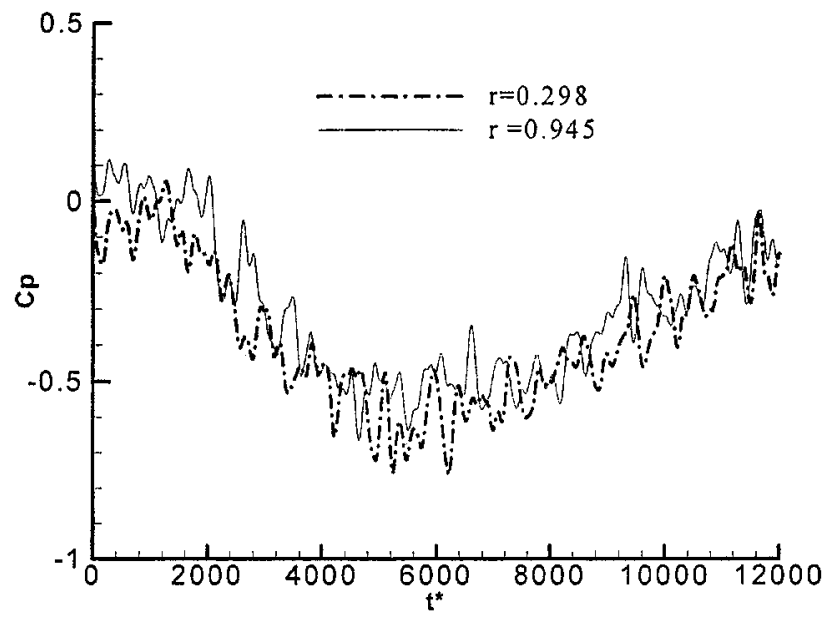

FIGURE 10

Unsteady pressure distributions on the suction side when fan starts up $(y / c=0.927)$. 


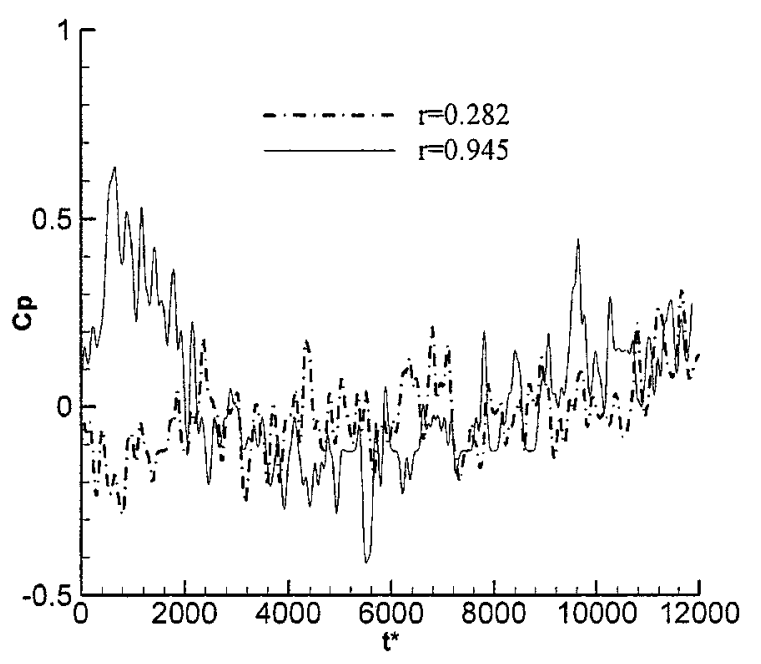

FIGURE 11

Unsteady pressure distributions on the pressure side when fan starts up $(y / c=0.071)$.

vibration, the unsteady pressure distribution measurements were conducted when the fan ran at the design condition.

The pressure variations with time on the suction surface for four different chordwise locations at two radial locations are shown in Figures 15-18. These results show that the pressure variations along the radial direction have similar behavior. The average periodical non-dimensional time of the pressure variation is about 28 , i.e. the frequency of the pressure variation is about $1 / 28$ of the fan rotational frequency. The pressure frequency can be calculated by using the unsteady Bernoulli's equation combined with Equations (2)-(5) (the detail calculation

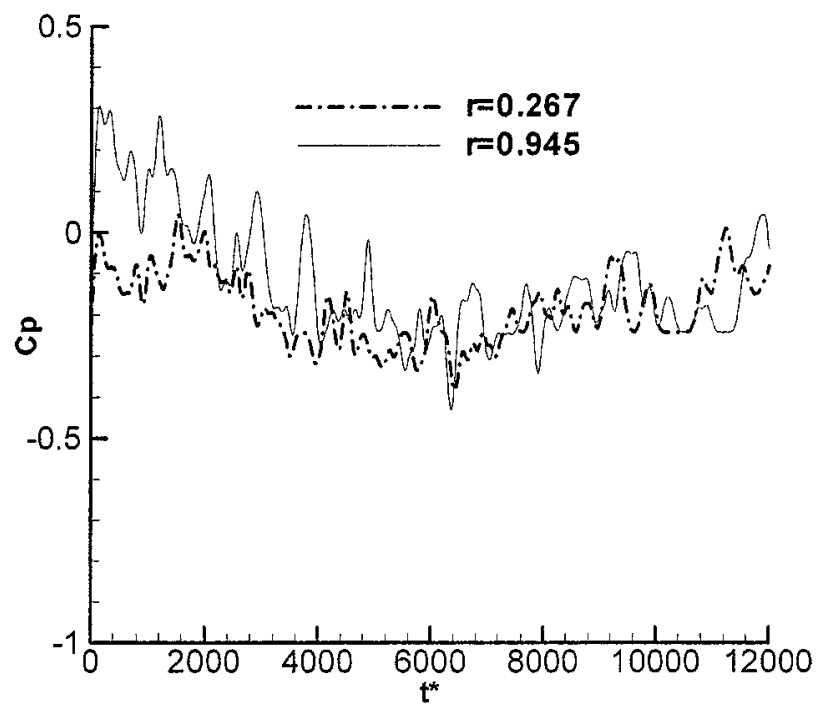

FIGURE 12

Unsteady pressure distributions on the pressure side when fan starts up $(y / c=0.304)$.

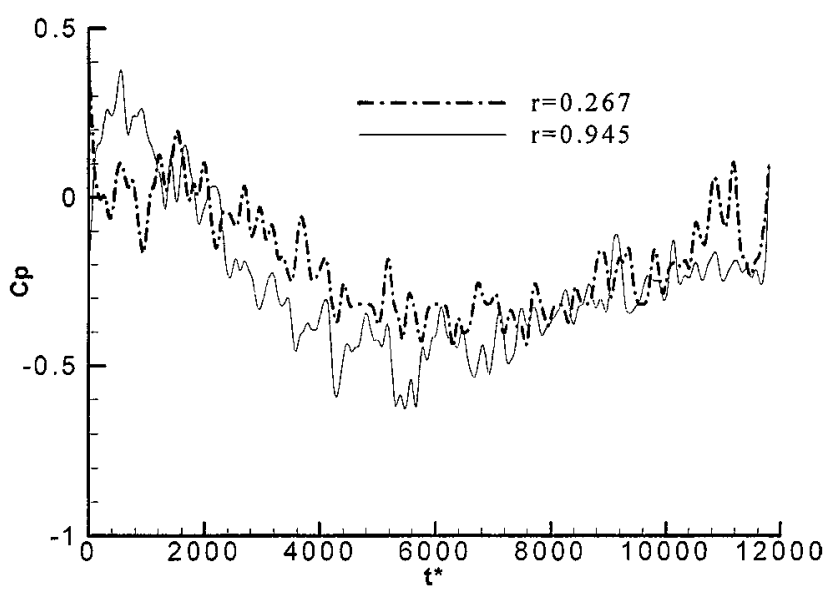

FIGURE 13

Unsteady pressure distributions on the pressure side when fan starts up $(y / c=0.611)$.

procedure can be found in Xu and Yeung, 1998). The pressure variation magnitude is smaller near the trailing edge, and it becomes larger in other chordwise locations. The theory without blade surface separation (Equation (11)) showed that the main frequencies of the velocity and pressure on the suction surface should have a similar value. Figure 16 shows that the frequency of pressure variation is larger than other chordwise locations. This is caused by the separation occurring on the suction surface near the chordwise location at $y / c=0.304$. This type of separation is not a bubble type separation; therefore it does not strongly influence the downstream pressure field. The influence of the separation on the pressure variation can only be limited

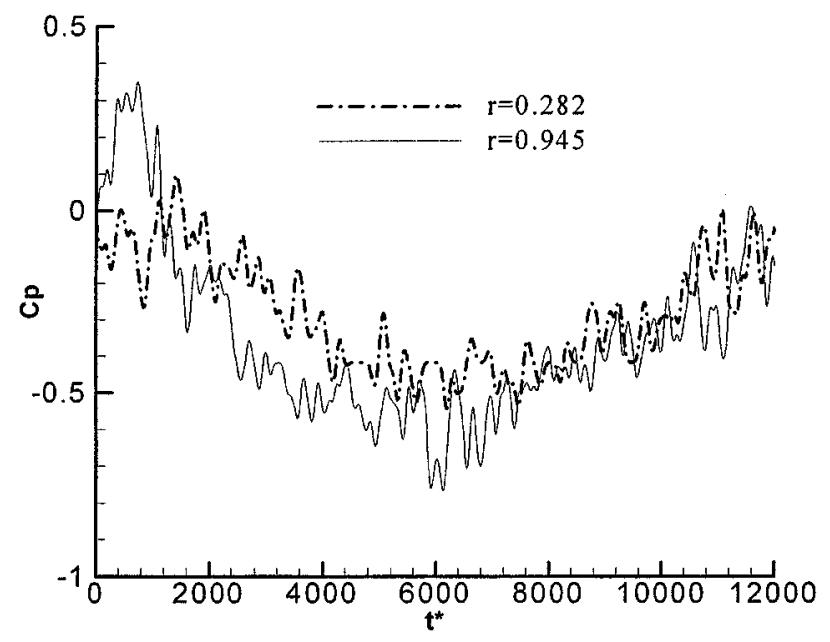

FIGURE 14

Unsteady pressure distributions on the pressure side when fan starts up $(y / c=0.0927)$. 


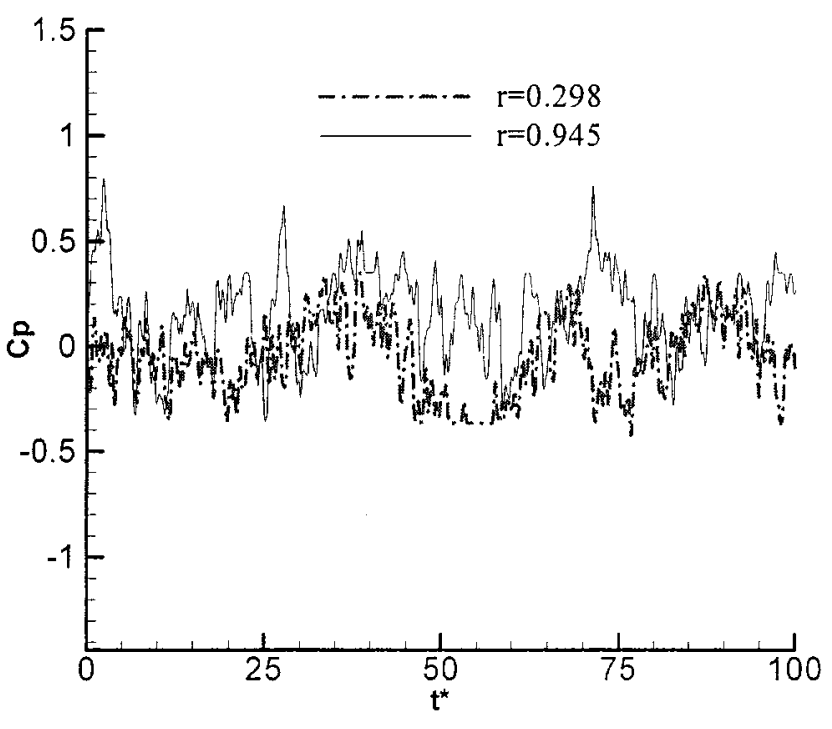

FIGURE 15

Unsteady pressure distributions on the suction side when fan runs at constant speed $(y / c=0.071)$.

near the separation region. This type of separation is very difficult to observe on the blade surface through flow visualization technology.

The pressure distributions on the pressure surface along the four different chordwise locations at two radial locations are shown in Figures 19-22. The pressure variations have similar frequencies on all locations which are caused by fan rotation. The periodical non-dimensional time of the pressure variation is about 28, which is almost the same as that on the pressure surface pressure. The average pressure levels for all locations generally show higher than those at the similar locations on the

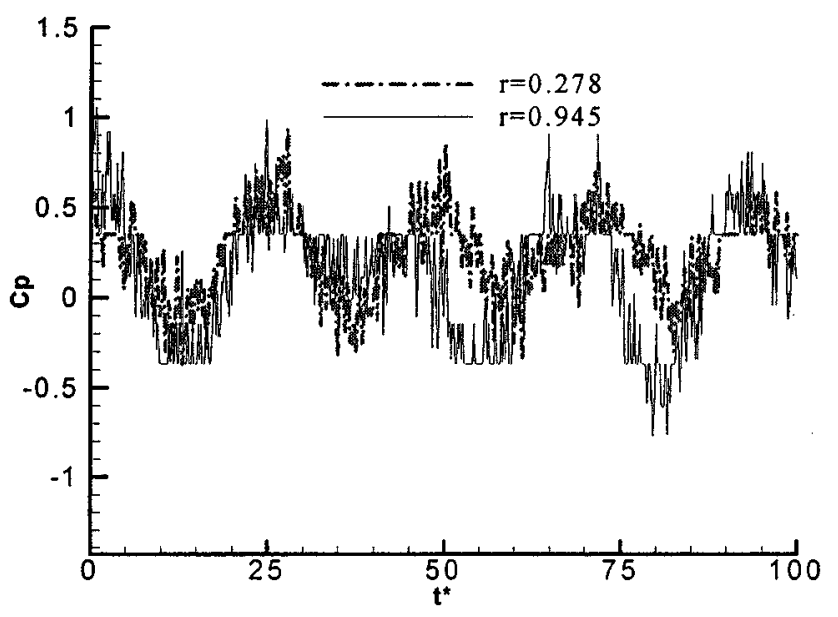

FIGURE 16

Unsteady pressure distributions on the suction side when fan runs at constant speed at $y / c=0.304$.

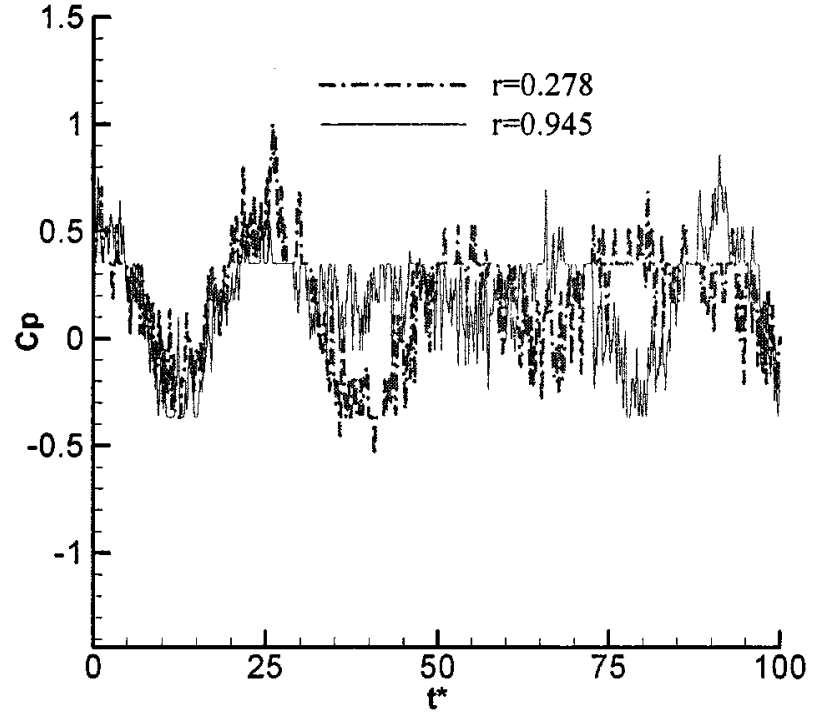

FIGURE 17

Unsteady pressure distributions on the suction side when fan runs at constant speed $(y / c=0.611)$.

suction surface. The oscillation magnitude is greater near the leading edge and lower near the trailing. This is because the flow has more oscillations near the leading edge than near the trailing edge. In general, the pressure oscillation magnitude on the pressure side is smaller than that on the suction side. This indicates that the turbulence level is smaller on the pressure side than on the suction side. It seems that all the blade surface pressure oscillations are determined only by the fan rotation.

To check the pressure variation due to shed vortices, the pressure variations near the radial center of blade are plotted in Figures 23 and 24 . The pressure distributions show that the

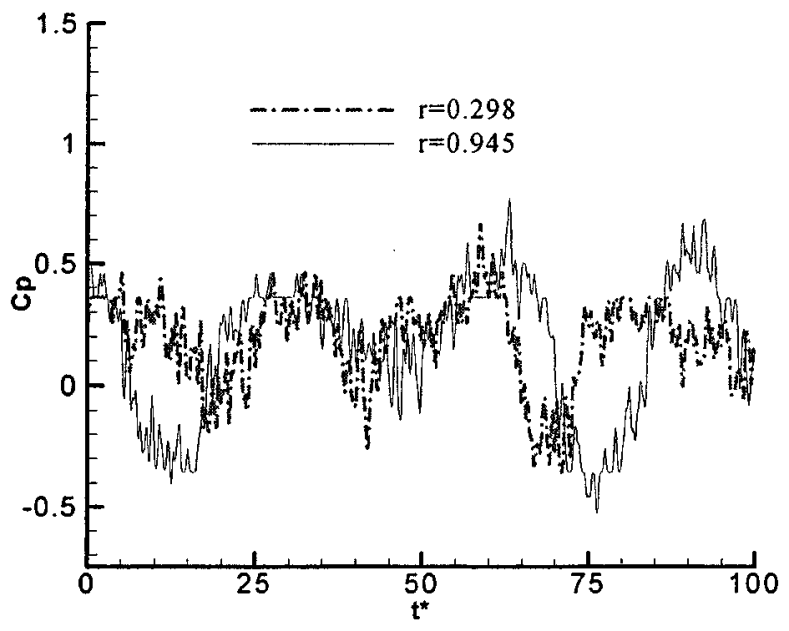

FIGURE 18

Unsteady pressure distributions on the suction side when fan runs at constant speed at $y / c=0.927$. 


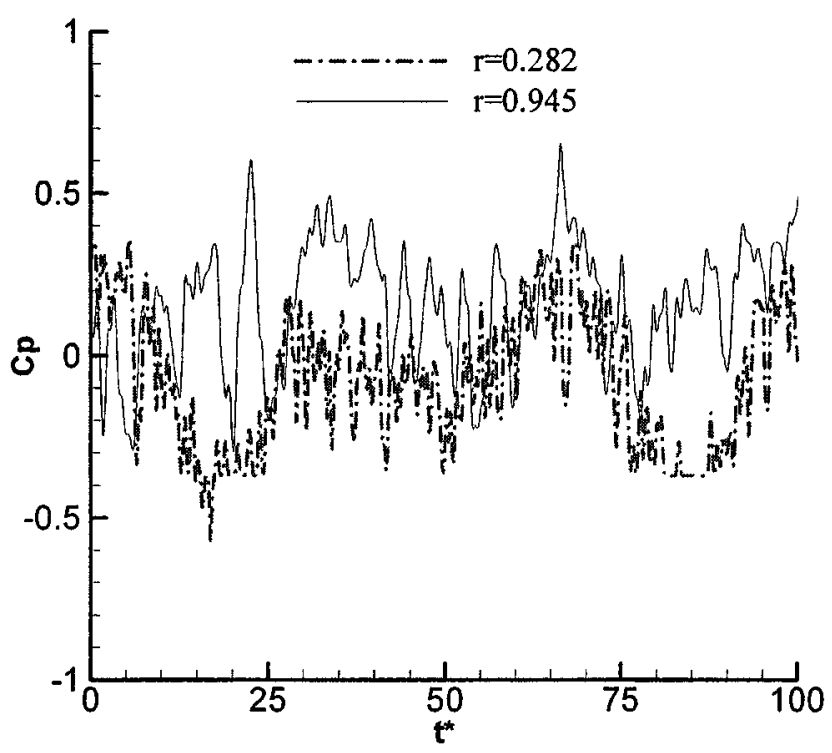

FIGURE 19

Unsteady pressure distributions on the pressure side when fan runs at constant speed $(y / c=0.0071)$.

pressure variations change not only with the fan rotation but also with the vortex shedding as indicated in Equations (2)-(5). Because the vortex shedding frequency is much higher than the frequency of rotation, the pressure variation frequency caused by the vortex shedding is also higher than those caused by the fan rotation in Figures 15-22 compared with Figures 23-24. Figures 23 and 24 show that the frequencies due to pressure variation are around 0.5 , which is about half of the fan rotational

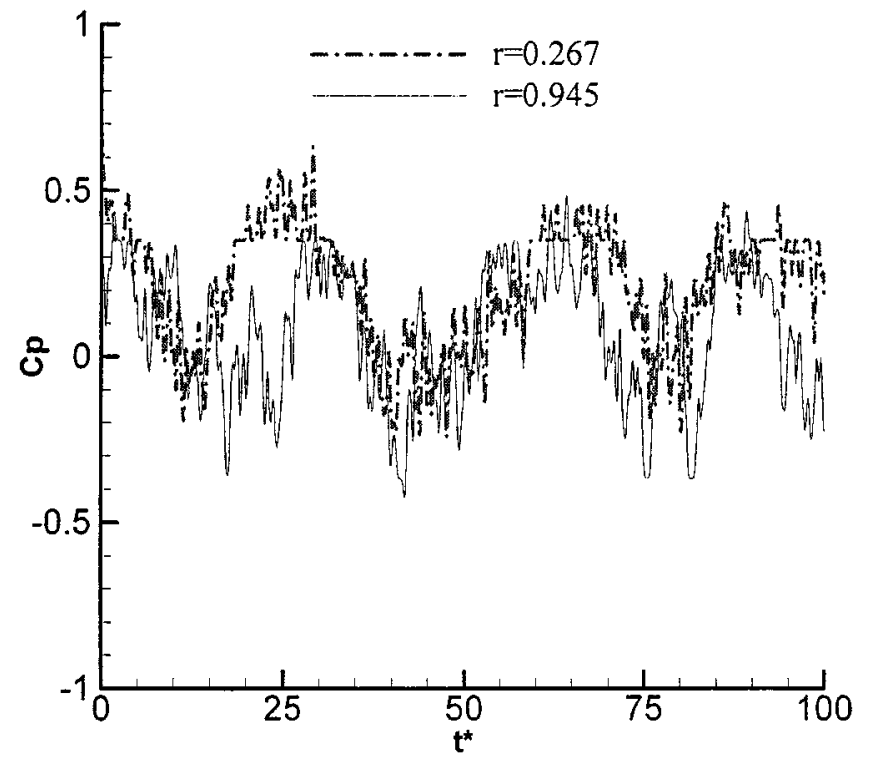

FIGURE 20

Unsteady pressure distributions on the pressure side when fan runs at constant speed at $y / c=0.304$.

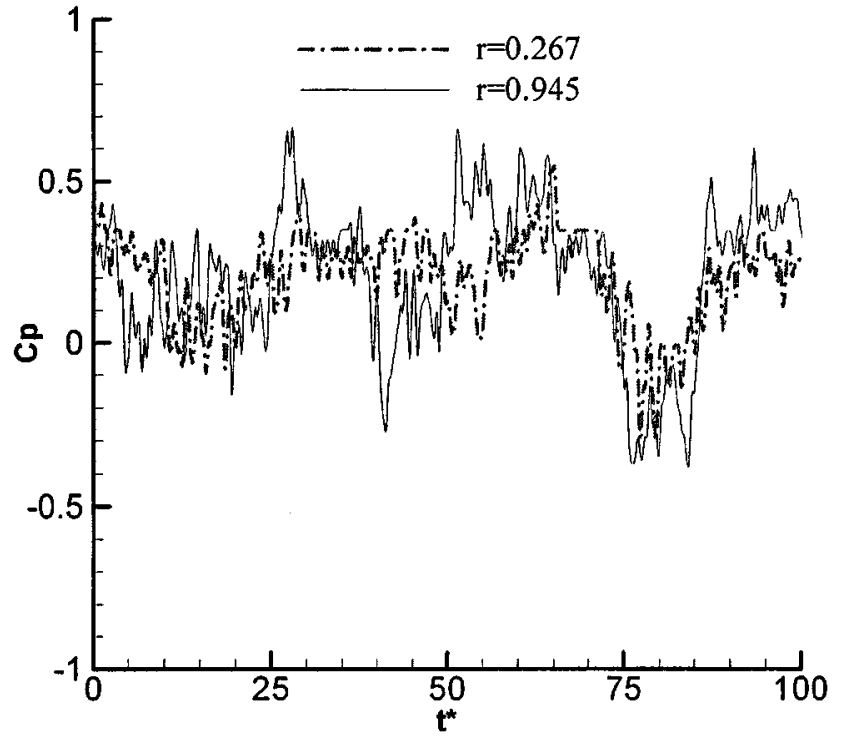

FIGURE 21

Unsteady pressure distributions on the pressure side when fan runs at constant speed $(y / c=0.611)$.

frequency. The magnitudes of the pressure variation with the vortex shedding are much lower than those with the fan rotation. Nonetheless, the frequency due to the pressure variation caused by the vortex is larger than the fan resonance frequency. This can be taken into consideration in improving the fan design. Figures 23-24 show that the pressure variations due to the vortex shedding have similar frequency both on the pressure and suction surfaces.

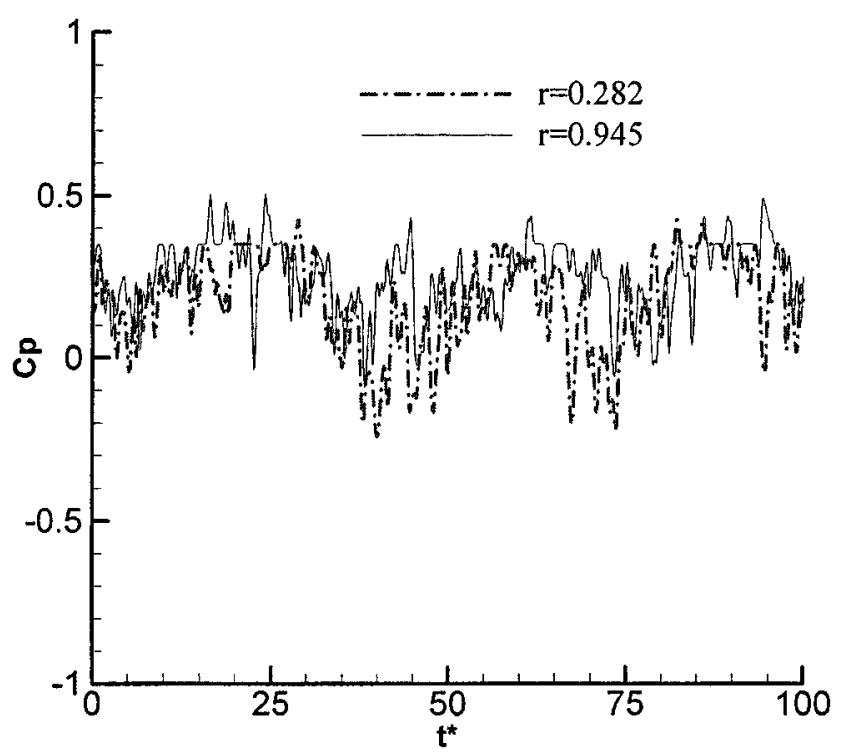

FIGURE 22

Unsteady pressure distributions on the pressure side when fan runs at constant speed $(y / c=0.927)$. 


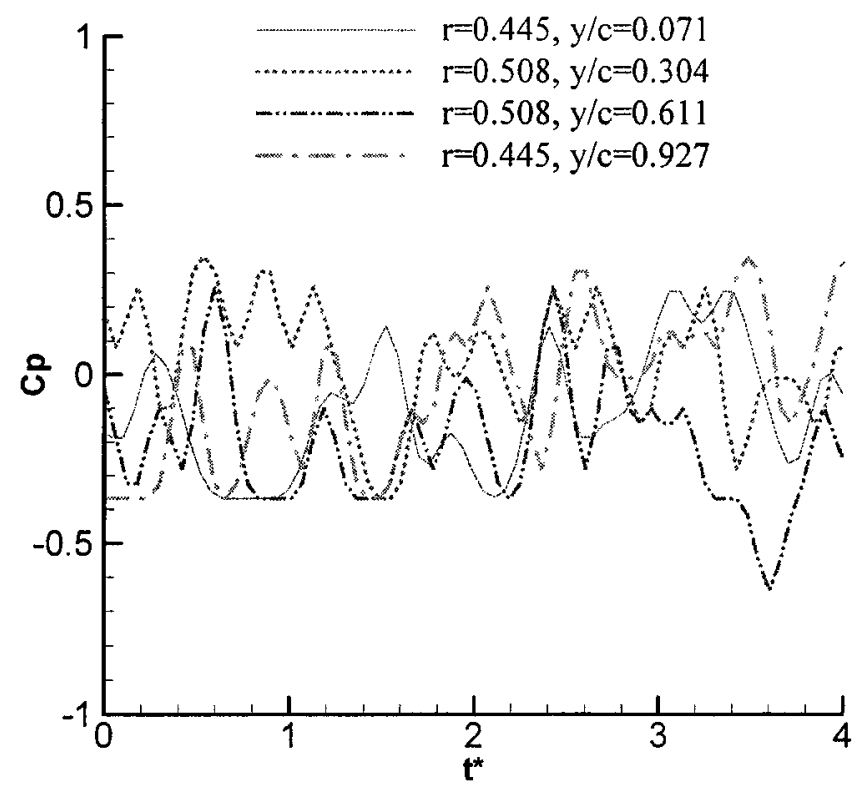

FIGURE 23

Unsteady pressure distributions on the pressure side.

The unsteady pressure measurements show that the flow fields near the blades are not completely steady, but rather are subject to flow field oscillations with time scales that are substantially longer than those associated with the blades.

\section{CONCLUSIONS}

In this study, a large flow-rate industrial axial fan was investigated. The flow visualizations and the unsteady pressure

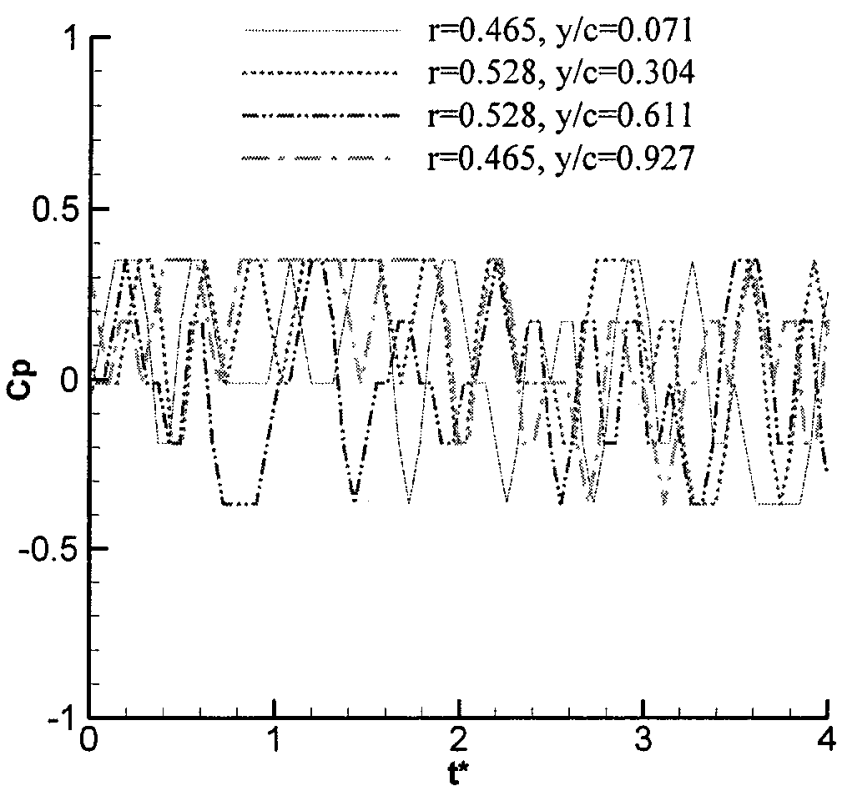

FIGURE 24

Unsteady pressure distributions on the pressure side. distributions on the fan blade were experimentally studied. The flow visualizations showed that the flow near the blade surface was strongly three-dimensional, with main flow direction varying from hub to tip. It was also observed that the flow near the blade surface was mainly influenced by the rotational centrifugal force and the stagger angle of the blade. The blade shape on the pressure side has little influence on the near-blade flow. Moreover, the flow acceleration on the suction side also has some influence on the near-blade flow.

The unsteady pressure measurement showed that the flow field produced by an axial fan is not completely steady. The frequency of the blade pressure distribution was related to the speed of the fan rotation, but is lower than the rotation speed.

The unsteady pressure measurements provide very important information about fan performance and vibration characteristics of the fan blades. The pressure variation database will provide a guide for a future fan design and the improvements of the present fan model. The reliable experimental results can be used as the database for computational modeling. Future studies should include the characterizations of the vibration and blade geometry aerodynamic influences.

\section{NOMENCLATURE}

$C$ chord of the fan blade

$C_{\mathrm{p}} \quad$ pressure coefficient

$d$ diameter of fan

$g \quad$ acceleration of gravity

$H$ total head

$l$

length of the casing

fan radius

$R \quad$ non-dimensional radius $=r / r_{t i p}$

$n \quad$ harmonic indices

$p \quad$ pressure

$p^{*} \quad$ total pressure

$s \quad$ coordinate along the streamline

$t \quad$ time

$t^{*} \quad$ non-dimensional time, $=t / T$

$T$ time of the rotation in one circle

$u \quad$ average velocity

$V \quad$ velocity

$V(s) \quad$ velocity at the vortex shedding point

$x$ axial distance from the rotational plan to test point non-dimensional axial distance $=x / r_{\text {tip }}$ non-dimensional chordwise coordinate number of blade

\section{Greek}

$\gamma \quad$ vortex strength

$\theta \quad$ stationary coordinate

$\tau \quad$ time defined by Eq. (7)

$\omega \quad$ shaft angular speed 


\section{Subscripts}

$b \quad$ blade or bounded vortex

$m \quad$ reference point

$i, j \quad$ indices of positions

$o \quad$ outlet plan

$q \quad$ indices

ref reference point

$s \quad$ steady state

$s 1, s 2$ leading and trailing edge

tip blade tip position

$w \quad$ wake

$\infty \quad$ infinite field or stagnation point

\section{REFERENCES}

Abramovich, G. N. 1963. The theory of turbulent jets. Cambridge, MA: MIT Press.

Capace, N. R., and Fleeter, S. 1989. Experimental Investigation of Multistage Interaction Gust Aerodynamics. ASME Journal of Engineering for Power 111(2):409-417.

Dring, R. P., Joslyn, H. D., Hardin, L. W., and Wagner, J. H. 1982. Turbine Rotor-Stator Interaction. ASME Journal of Engineering for Power 104(3):729-742.

Du, H., Han, J. C., and Ekkad, S. V. 1998. Effect of Unsteady Wake on Detailed Heat Transfer Coefficient and Film Effectiveness Distributions for a Gas Turbine Blade. ASME Journal Turbomachnery 120(4):808-817.

Gallus, H. E., Lambertz, J., and Wallmann, T. 1980. Blade-Row Interaction in an Axial-Flow Subsonic Compressor Stage. ASME Journal of Engineering for Power 102(1):169-177.

Huntsman, I., and Hodson, H. P. 1995. Laminar Flow Rotor for a Radial Inflow Turbine. Journal of Propulsion and Power 11(5):1170 1178 .
Huyer, S. A., Simms, D., and Robinson, M. C. 1996. Unsteady aerodynamics associated with a horizontal-axis wind turbine. AIAA Journal 34(7):1410-1419.

Kaupert, K. A., and Staubli, T. 1999a. The unsteady pressure field in a high specific speed centrifugal pump impeller-Part 1: Influence of the volute. ASME Journal of Fluids Engineering 121(3):621-626.

Kaupert, K. A., and Staubli, T. 1999b. The unsteady pressure field in a high specific speed centrifugal pump impeller-Part 2: Transient hysteresis in the characteristic. ASME Journal of Fluids Engineering 121(3):667-632.

Matlab Signal Processing Toolbox User's Guide Ver. 4.0. 1996, Natick, MA: math Works.

Minniti, R. J., Sullivan, C. J., and Mueller, T. J. 1998. A thin film sensor for the measurement of unsteady pressure. The Aeronautical Journal 102(1017):393-398.

Mutama, K. R., and Hall, A. E. 1996. The experimental investigation of jet fan aerodynamics using wind tunnel modeling. ASME Journal of Fluids Engineering 118(2):322-327.

Myers, K. J., Ward, R. W., and Bakker, A. 1997. A digital particle image velocimetry investigation of flow field instabilities of axialflow impellers. Journal of Fluids Engineering 119(3):623-632.

Tsurusaki, H., Tsujimoto, Y., Yoshida, Y., and Kitagawa, K. 1997. Visualization measurement and numerical analysis of internal flow in cross-flow fan. Journal of Fluids Engineering 119(3):633-638.

$\mathrm{Xu}, \mathrm{C}$., and Amano, R. S. 1999. The nonsymmetrical uncertainty intervals in fluid measurement system. Mechanical Engineering Research Report. Milwaukee: University of Wisconsin-Milwaukee.

$\mathrm{Xu}, \mathrm{C}$., and Amano, R. S. 2000. The nonsymmetrical uncertainty intervals in fluid measurement system. Mechanical Engineering Research Report. Milwaukee: University of WisconsinMilwaukee.

Xu, C., and Yeung, W. W. H. 1998. Numerical study of unsteady flow around airfoil with spoiler. ASME Journal of Applied Mechanics 65(1):164-170. 

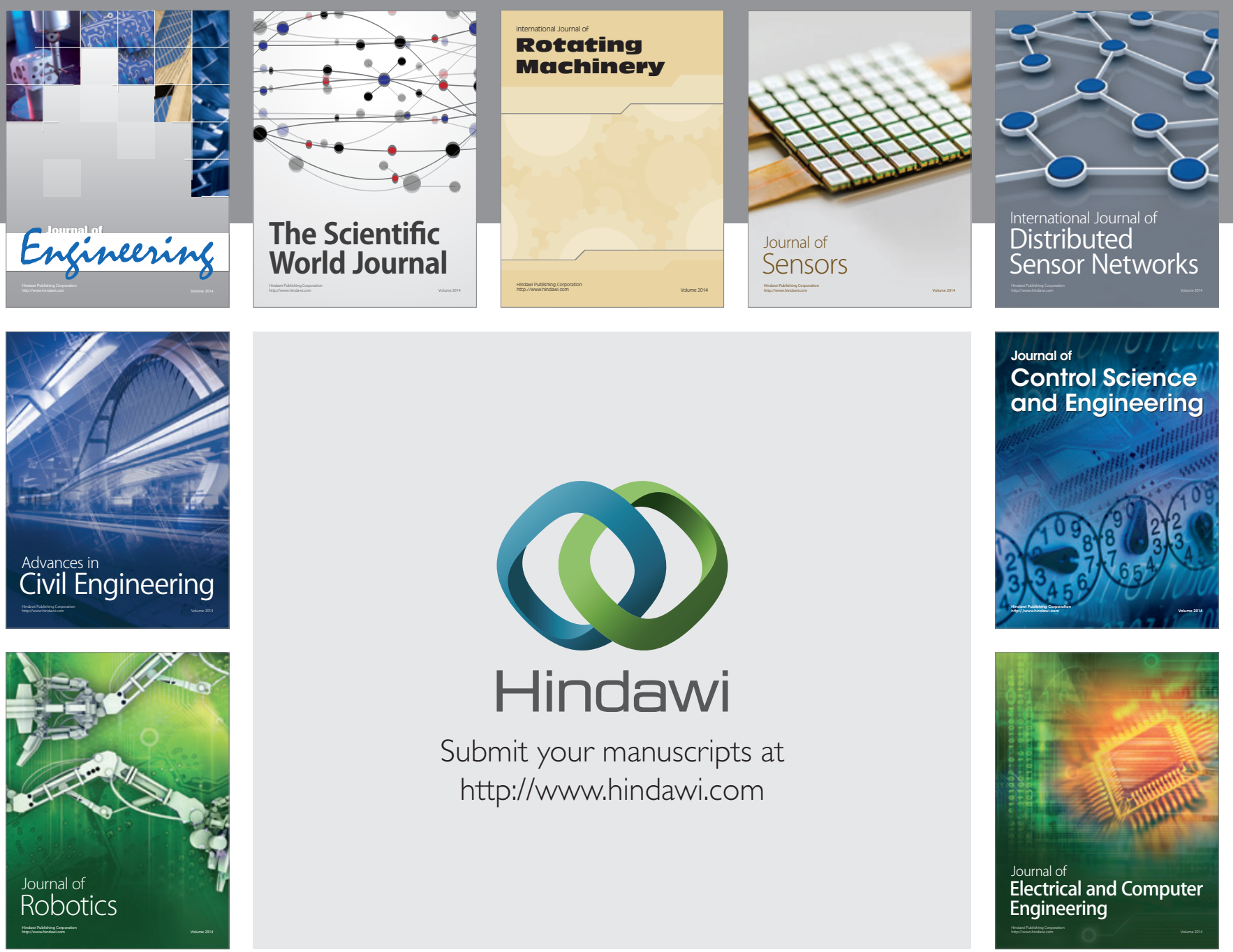

Submit your manuscripts at

http://www.hindawi.com
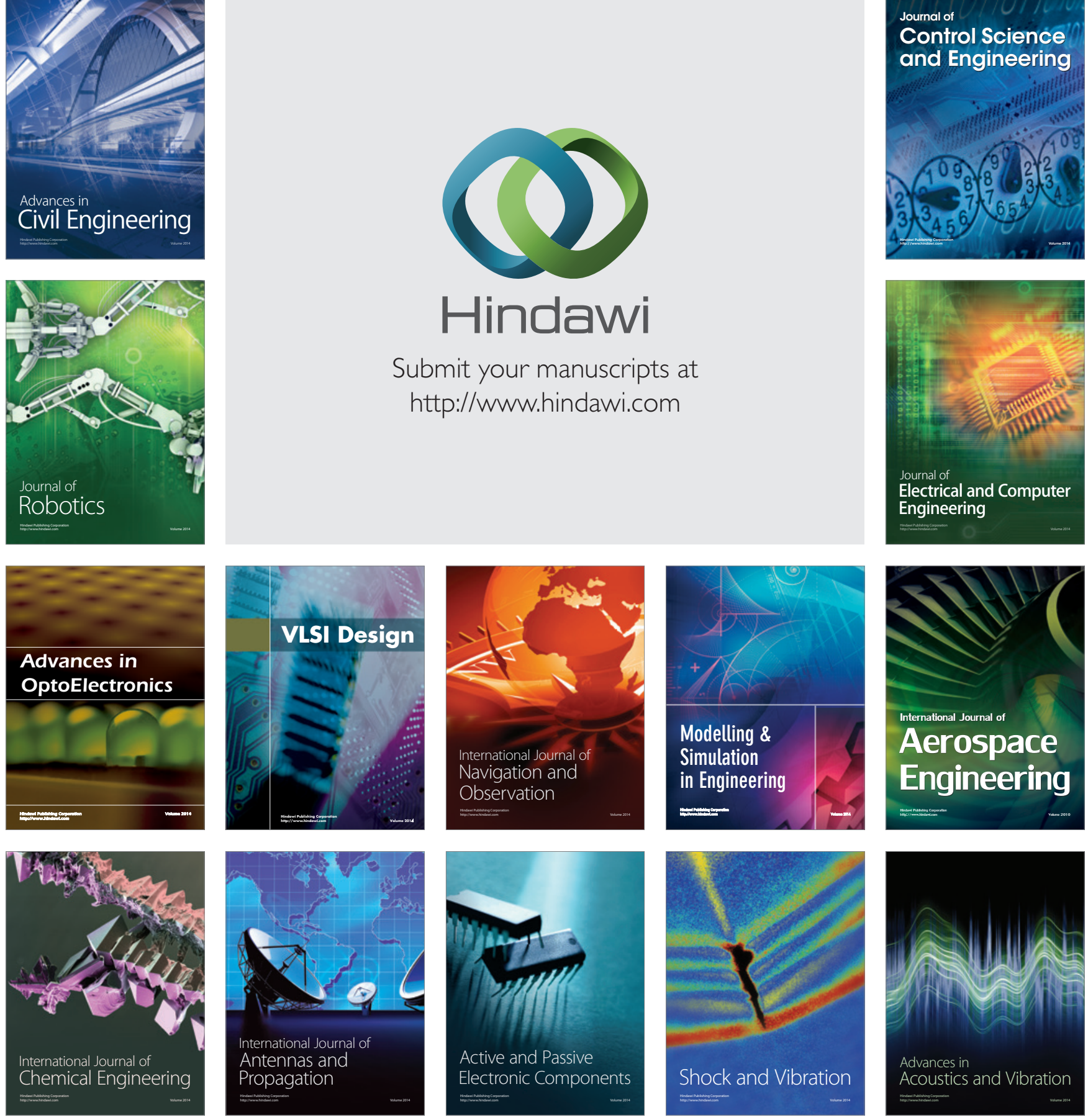